\title{
Susceptibility to mycobacterial disease due to mutations in IL-12R $\beta 1$ in three Iranian patients
}

\author{
Maryam Alinejad Dizaj ${ }^{1,2} \cdot$ Esmaeil Mortaz $^{3,4} \cdot$ Seyed Alireza Mahdaviani ${ }^{5}$. \\ Davood Mansouri $^{3} \cdot$ Payam Mehrian $^{2} \cdot$ Els M. Verhard ${ }^{6}$ • Mohammad Varahram ${ }^{7}$. \\ Delara Babaie $^{8}$ - Ian M. Adcock ${ }^{9,10}$ • Johan Garssen ${ }^{4,11}$ • Esther van de Vosse ${ }^{6}$. \\ Aliakbar Velayati ${ }^{7}$
}

Received: 2 September 2017 / Accepted: 26 October 2017 /Published online: 18 December 2017

(C) The Author(s) 2017. This article is an open access publication

\begin{abstract}
In the last decade, autosomal recessive interleukin12 receptor $\beta 1$ (IL-12R $\beta 1$ ) deficiency, the most common cause of Mendelian susceptibility to mycobacterial disease (MSMD), has been diagnosed in a few children and adults with severe tuberculosis in Iran. Here, we report three cases referred to the Immunology, Asthma and Allergy ward at the National Research Institute of Tuberculosis and Lung Diseases (NRITLD) at Masih Daneshvari Hospital from 2012 to 2017 with Mycobacterium tuberculosis and nontuberculous mycobacteria infections due to defects in IL$12 \mathrm{R} \beta 1$ but with different clinical manifestations. All three were homozygous for either an IL-12R $\beta 1$ missense or nonsense mutation that caused the IL- $12 \mathrm{R} \beta 1$ protein not to be expressed on the cell membrane and completely abolished the cellular response to recombinant IL-12. Our findings suggest that the presence of IL-12R $\beta 1$ deficiency should be
\end{abstract}

Esmaeil Mortaz

Emortaz@gmail.com

1 Department of Biotechnology, School of Advanced Technologies in Medicine, Shahid Beheshti University of Medical Sciences, Tehran, Iran

2 Chronic Respiratory Diseases Research Center, National Research Institute of Tuberculosis and Lung Diseases (NRITLD), Shahid Beheshti University of Medical Sciences, Tehran, Iran

3 Clinical Tuberculosis and Epidemiology Research Center, National Research Institute of Tuberculosis and Lung Diseases (NRITLD), Shahid Beheshti University of Medical Sciences, Tehran, Iran

4 Division of Pharmacology, Utrecht Institute for Pharmaceutical Sciences, Faculty of Science, Utrecht University, Utrecht, The Netherlands

5 Pediatric Respiratory Diseases Research Center, National Research Institute of Tuberculosis and Lung Diseases (NRITLD), Shahid Beheshti University of Medical Sciences, Tehran, Iran determined in children with mycobacterial infections at least in countries with a high prevalence of parental consanguinity and in areas endemic for TB like Iran.

Keywords IFN- $\gamma \cdot \mathrm{IL} 12 \mathrm{RB} 1 \cdot \mathrm{IL}-12 \mathrm{R} \beta 1 \cdot \mathrm{IMD} 30 \cdot$ MSMD · PID

\section{Introduction}

Natural human immunity to intracellular pathogens, including opportunistic or low-virulence mycobacteria such as Bacillus Calmette-Guérin (BCG; an attenuated Mycobacterium bovis strain) vaccines, non-tuberculous mycobacteria (NTM) and non-typhoid Salmonella, and/or Mycobacterium tuberculosis (MTB), relies on the functional integrity of the interleukin (IL)

6 Department of Infectious Diseases, Leiden University Medical Center, Leiden, The Netherlands

7 Mycobacteriology Research Center, National Research Institute of Tuberculosis and Lung Diseases (NRITLD), Shahid Beheshti University of Medical Sciences, Tehran, Iran

8 Department of Allergy and Clinical Immunology, Mofid Children's Hospital, Shahid Beheshti University of Medical Sciences, Tehran, Iran

9 Cell and Molecular Biology Group, Airways Disease Section, National Heart and Lung Institute, Imperial College London, Dovehouse Street, London, UK

10 Priority Research Centre for Healthy Lungs, Hunter Medical Research Institute, The University of Newcastle, Newcastle, New South Wales, Australia

11 Nutricia Research Centre for Specialized Nutrition, Utrecht, The Netherlands 
12/23-interferon (IFN)- $\gamma$ axis enabling cross-talk between macrophages and T-lymphocytes/NK cells (Lee et al. 2011; Mortaz et al. 2015; Prando et al. 2013). Inborn errors of immunity (primary immunodeficiency) such as Mendelian susceptibility to mycobacterial disease (MSMD) emphasize the critical role of this axis in the defense against these pathogens (Casanova and Abel 2002; Feinberg et al. 2004; Haverkamp et al. 2014; Serour et al. 2007).

MSMD-causing mutations have been identified in eight autosomal genes (IFNGR1, IFNGR2, STAT1, IL12B, $I L 12 R B 1, I R F 8, I S G 15$, and TYK2) and two X-linked genes (IKBKG and CYBB) (Bogunovic et al. 2012; Bustamante et al. 2011; Filipe-Santos et al. 2006; Hambleton et al. 2011; Haverkamp et al. 2014; Prando et al. 2013) resulting in impaired IFN- $\gamma$-mediated immunity (Casanova and Abel 2002; de Beaucoudrey et al. 2010).

IFN- $\gamma$ is essential for killing and controlling mycobacterial infections (Casanova and Abel 2002; Lee et al. 2011). Complete deficiency of either of the two IFN- $\gamma$ receptor chains (IFN- $\gamma \mathrm{R} 1$ and IFN- $\gamma \mathrm{R} 2$ ) or signal transducer and activator of transcription (STAT)-1 is associated with the development of disseminated infection in early childhood, has a very poor prognosis, and is often fatal. Complete IL- $12 p 40$ and IL-12R $\beta 1$ deficiencies, as well as partial IFN- $\gamma$ R1, IFN- $\gamma$ R2, and STAT1 deficiencies, are generally associated with a later onset disease, milder clinical infections, and a good prognosis (Altare et al. 2001; Casanova and Abel 2002; Casanova et al. 2002; Ottenhoff et al. 2002; Özbek et al. 2005; Reichenbach et al. 2001).

Genetic analysis of a large cohort of 220 MSMD patients with a defective IL-12/IL-23-IFN- $\gamma$ axis ranked the presence of these mutations among MSMD patients as IL12RB1 (40\%), IFNGR1 (39\%), IL12B (9\%), STAT1 (5\%), IFNGR2 (4\%), and IKBKG (3\%) (Casanova et al. 1995; Filipe-Santos et al. 2006; Lee et al. 2011). In addition, IL-12R $\beta 1$ deficiency was the first primary immunodeficiency to be associated with pediatric tuberculosis in children with normal resistance to BCG and environmental mycobacteria (EM) (de Beaucoudrey et al. 2010). IL-12R $\beta 1$ deficiency, therefore, should probably be suspected in any patient with an unusual infection with intracellular pathogens even in the absence of parental consanguinity.

IL-12R $\beta 1$ deficiency has been diagnosed in several children and teenagers with tuberculosis (TB). The prevalence of TB in IL-12p40- and IL-12R $\beta 1$-deficient patients is however lower than BCG or NTM infection (Caragol et al. 2003; de Beaucoudrey et al. 2010; Özbek et al. 2005). Until 2005, only $4 / 73(5.5 \%)$ patients with IL-12R $\beta 1$ or IL-12p40 deficiency had been reported to have TB $(3 / 54$ patients with complete IL$12 \mathrm{R} \beta 1$ deficiency and 1/19 patients with complete IL-12p40 deficiency) (Fieschi and Casanova 2003; Özbek et al. 2005). BCG vaccination, and especially developing BCG disease, confers a greater protection against EM disease than against TB in IL-12R $\beta 1$-deficient patients despite the close phylogenetic relationship between BCG and MTB, presumably because MTB is more virulent than EM (de Beaucoudrey et al. 2010).

Disseminated disease in children and pulmonary disease in adults constitute two major epidemiological and clinical forms of TB infection (Alcaïs et al. 2005). Understanding the causes of mycobacterial infection in children and adults and finding the driver mutations in the IL-12/IL-23-IFN- $\gamma$ axis may provide a new approach for controlling the tuberculosis infections. Here, we report three MSMD patients from Iran with MTB and NTM infection due to a complete inability to produce IFN- $\gamma$ in response to IL-12 due to mutations in IL-12R $\beta 1$.

\section{Case reports}

The three children were either born to consanguineous parents (patients 1 and 3) or to unrelated parents (patient 2) and were referred to the Immunology, Asthma and Allergy ward at the National Research Institute of Tuberculosis and Lung Diseases (NRITLD) at Masih Daneshvari Hospitals, Shahid Beheshti University of Medical Sciences, Tehran-Iran, between January 2012 and July 2017.

\section{Patient 1}

This was an 8-year-old female born to consanguineous parents from the center of Iran (Table 1; patient 1). She was vaccinated with live attenuated Mycobacterium bovis BCG sub-strain Pasteur at birth. Two months later, she developed a progressive bilateral axillary adenopathy (Fig. 1a) but was discharged without any medical intervention. At the age of 6 months, the patient developed supraclavicular lymphadenopathy. MTB complex was detected in drainage secretions and anti-TB medication was initiated and continued for 6 months.

Two years later, the patient was hospitalized due to high blood pressure, pyrexia, myalgia, cough, and severe weight loss. Evaluation of gastric washing (GW) cultures, smeardetected Mycobacterium, acid fast bacilli (AFB), and a polymerase chain reaction (PCR) test was positive for the MTB complex and BCG. Chest radiography showed right-sided pleural effusion and adenopathy in the para-aortic, celiac, and mesenteric regions was seen. Both kidneys were normal in size although evidence of abscesses on the left side was observed by ultrasound. PCR tests for urine and pleural fluid were also positive for BCG. An anti-TB treatment course was started with isoniazid, rifampin, ethambutol, Klacid, ofloxacin, and Lanoxin. Two months later, despite a negative PCR result for the MTB complex in urine and GW, the previous treatment regimen was complemented with clarithromycin, captopril, and IFN- $\gamma\left(25 \mu \mathrm{g} / \mathrm{m}^{2}\right)$. A few months later, AFB was isolated following bone marrow (BM) aspiration and an immunodeficiency was suspected. 
Table 1 Genotypes and clinical phenotypes of patients with IL-12R $\beta 1$ deficiency

\begin{tabular}{llllll}
\hline Patient no. & Age $/ \mathrm{sex}$ & Age of onset (months) & $\begin{array}{l}\text { Kind of mycobacterial } \\
\text { infection }\end{array}$ & $\begin{array}{l}\text { Clinical manifestations } \\
\text { after BCG vaccination }\end{array}$ & Involved exon/mutation \\
\hline 1 & $8.3 / \mathrm{F}$ & 3 & BCG & Bilateral axillary adenopathy & Exon 5/c.512A $>$ C, Q171P \\
2 & $3.7 / \mathrm{M}$ & 3 & BCG & Left axillary lymphadenopathy & Exon 9/c.847C $>$ T, R283X \\
3 & $7.3 / \mathrm{M}$ & 5 & BCG and MTB & Axillary lymphadenopathy & Exon 5/c.517C $>$ T, R173W \\
\hline
\end{tabular}

BCG Bacille-Calmette-Guérin, MTB Mycobacterium tuberculosis

${ }^{a}$ Age at death, last follow-up, or at the time of writing this report

Eight months later, clinical manifestations (persistent cough and dyspnea) continued to be reported despite treatment and the patient was admitted to the hospital. Various mycobacteriologic analyses (including sputum smears and cultures, drug susceptibility test (DST), PCR, and other tests) were performed. GW PCR showed the presence of MTB complex that was isoniazid- and rifampin-resistant. Blood tests showed a WBC of $6.1 \times 10^{3} / \mu 1($ Neu $71 \%$; Lymph $22 \%$; Mix 7\%); $\mathrm{Hb} 10.6 \mathrm{~g} / \mathrm{dl}$; HCT33\%; and the ESR was $39 \mathrm{~mm} / \mathrm{h}$ in the first hours with detected high C-reactive protein (CRP) $(78 \mathrm{mg} / \mathrm{l}$; normal range $<10 \mathrm{mg} / \mathrm{l})$. Liver function tests revealed an aspartate aminotransferase (AST) of $36 \mathrm{u} / 1$ (normal 15-50 u/l), alanine aminotransferase (ALT) of $10 \mathrm{u} / 1$ (normal 10-25 u/l), and a decreased ALT/AST ratio. Antibody responses to tetanus and diphtheria were normal and DHR123 and nitroblue- tetrazolium (NBT) tests were within the normal range. The patient was treated again with amikacin, Klacid, cycloserine, Lanoxin, captopril, and IFN- $\gamma\left(25 \mu \mathrm{g} / \mathrm{m}^{2}\right)$. After a few months of therapy, whole blood was collected from both the patient and her parents and the release of IL-12 and IFN- $\gamma$ from stimulated peripheral blood mononuclear cells (PBMCs) was assessed. In brief, PBMCs were isolated from whole blood containing EDTA using Ficoll-Paque before being stimulated with rhIL-12 (Gibco, Life Technologies, USA; $20 \mathrm{ng} / \mathrm{ml}$ ), rhIFN- $\gamma$ (Gibco, Life Technologies, USA; 5000 UI/ $\mathrm{ml}$ ), and/or BCG for $48 \mathrm{~h}$ and IFN- $\gamma$ and IL-12 release was measured by ELISA.

IFN- $\gamma$ release following stimulation with BCG + rhIL12 was extremely low and a 100 -fold lower than that observed from PBMCs from control subjects. Although the addition of rhIFN- $\gamma$ to BCG-stimulated cells further
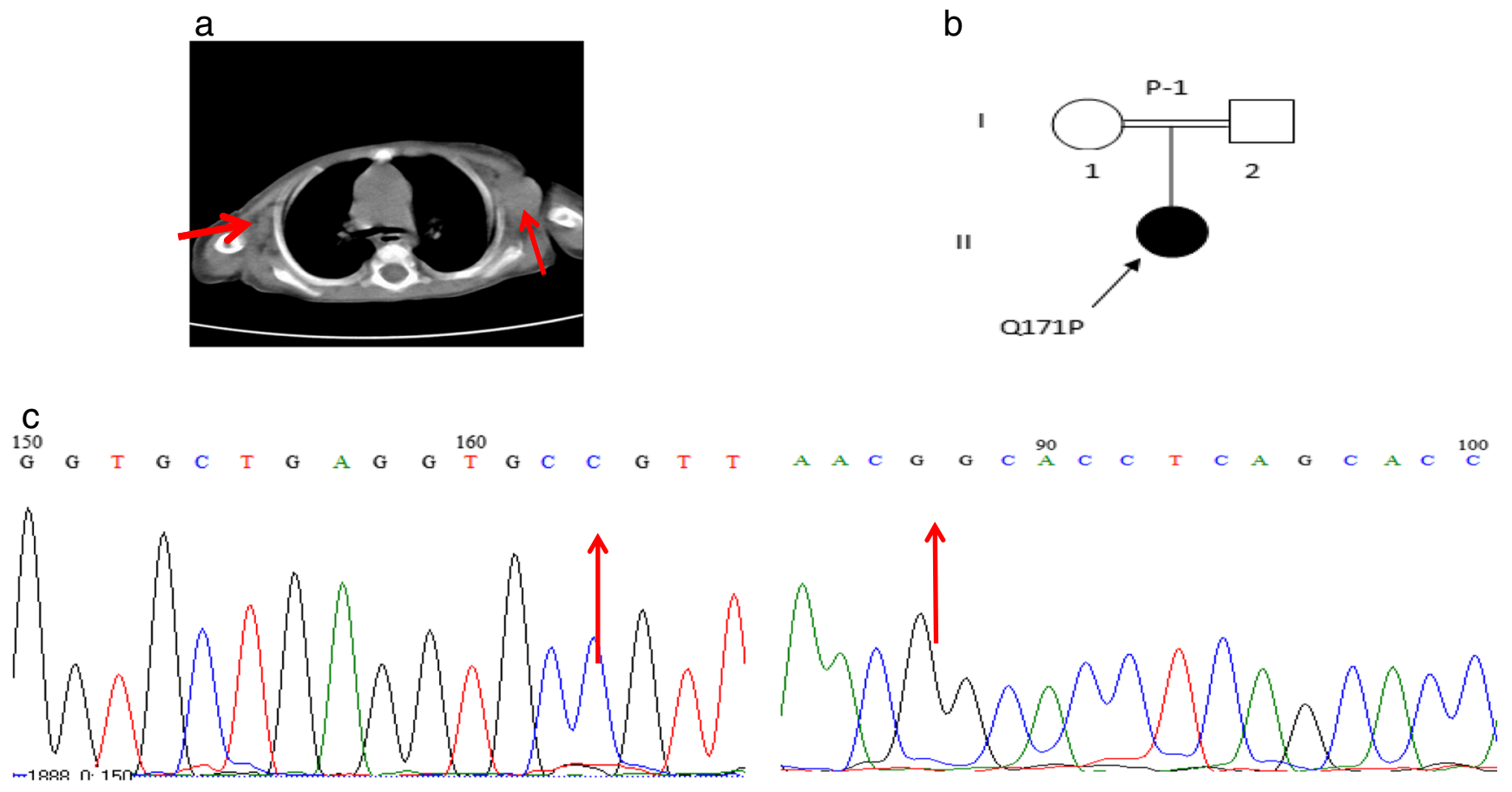

Fig. 1 Patient 1. a CT scan without contrast. Note bilateral axillary adenopathies (red arrow). b Pedigree of patient 1 . c Sequencing analysis by dideoxynucleotide termination method demonstrated a mutation in exon 5 of the $I L-12 R B 1$ gene using forward (left panel) and reverse (right panel) sequencing 
increased IL-12p70 release from PBMCs 4.4-fold, this remained far less than that seen from normal healthy controls (Table 2; patient 1) and was similar to that reported in various MSMD patients (Feinberg et al. 2004). These functional data indicated a possible genetic defect in IL$12 \mathrm{R} \beta 1$.

To examine this, genomic DNA was isolated from PBMCs by salt extraction and the IL-12R $\beta 1$ coding exons and flanking regions of the introns were amplified by PCR and sequenced at Leiden University Medical Center and a homozygous mutation in IL12RBI exon 5, c. $512 \mathrm{~A}>\mathrm{C}$, which results in the amino acid substitution p.Q171P was identified (Fig. 1c). Both parents were heterozygous for this mutation. This mutation has been reported previously to result in complete lack of IL-12R $\beta 1$ protein expression on the cell membrane (Fieschi et al. 2003). To our knowledge, this is the first case from Iran with this mutation.

\section{Patient 2}

The second case, a male born to unrelated parents and vaccinated with BCG at birth, was diagnosed with left axillary lymphadenopathy at the age of 3 months (Table 1; patient 2). Three months later, the patient developed bilateral inguinal lymphadenopathy. This was drained and the patient discharged. The patient was diagnosed with BCG-osis and treated with anti-TB drugs (rifampin, isoniazid, cycloserin, IFN- $\gamma$ and, ethambutol), but despite this treatment, the patient was re-admitted to the hospital at the age of 7 months complaining of respiratory distress due to isoniazid use. Histological analysis of a cervical lymph node biopsy revealed proliferating activated histiocytes and macrophages, inflammatory cell infiltration, and a few scattered giant cells. PCR analysis of the lymph node biopsy was positive for the MTB complex and BCG but negative for viral infections including EBV and CMV. On physical examination, the patient had hepatosplenomegaly, lymphadenopathy in the para-aortic region and mesenteric roots, and bilateral axillary adenopathy. Treatment was continued for nearly 2 years but the patient's general condition continued to deteriorate despite anti-TB treatment and he was again admitted to the hospital complaining of severe backache and abdominal ascites. Blood analysis gave the following results: ESR $11 \mathrm{~mm} / \mathrm{h}$, WBC $4.1 \times 10^{3} / \mu \mathrm{l}$ (Neu 39.6\%; Lymph 43.7\%; Mix 16.7\%), $\mathrm{Hb} 11.9 \mathrm{~g} / \mathrm{dl}$, PLT $120 \times 10^{3} / \mu \mathrm{l}$ with a low liver function test ALT/AST ratio (310/345), and high alkaline phosphatase (ALP) $3400 \mathrm{u} / \mathrm{ml}$ (normal range $180-1200 \mathrm{u} / \mathrm{ml}$ ). A qualitative C-reactive protein test was positive. An evaluation of the abdominal cavity by imaging and by physical examination showed moderate abdominal ascites and splenomegaly $(116 \mathrm{~mm})$. Liver biopsy showed chronic hepatitis, grade $3 / 18$, stage $2 / 6$. This condition is seen in both viral and autoimmune hepatitises. Plasma cell infiltration, lymphoid aggregates, or follicles (seen in autoimmune hepatitis) were absent and both liver biopsy and ascites were negative for AFB and mycobacteria. An MSMD functional assay was conducted in whole blood (PBMCs) and revealed a lack of response to recombinant IL-12 following BCG stimulation (Table 2; patient 2). Genomic sequence analysis revealed a c. $847 \mathrm{C}>\mathrm{T}$ mutation in exon 9 of the IL12RB1 gene (Fig. 2b). This defect results in a change from R283 to a premature stop codon (p.R283X). Both parents were heterozygous for this mutation. This variation has been previously reported in a single patient from the Faroe Islands (Schejbel et al. 2011) and in two patients from Brazil (Falcão et al. 2012). Despite anti-TB and broad-spectrum antibiotic treatment, the patient's general condition continued to deteriorate and the patient died a year after the detection of his immunodeficiency status at the age of 3 years and 7 months.
Table 2 In vitro production of IFN- $\gamma$ and IL-12p70 following stimulation in three IL-12R $\beta 1$ deficient patients and healthy control subjects

\begin{tabular}{llcccc}
\hline Cytokine detected & Stimulus $^{\mathrm{a}}$ & Patient 1 & Patient 2 & Patient 3 & $\begin{array}{c}\text { Healthy controls } \\
\text { Mean } \pm \text { SEM } \\
\end{array}$ \\
& & & & $n=6$ \\
\hline IFN- $\gamma(\mathrm{pg} / \mathrm{ml})$ & Medium & 0 & 0 & 1.8 & $37.4 \pm 12.4$ \\
& BCG & 47.2 & 15.4 & 35.04 & $1059.9 \pm 136.9$ \\
& BCG + rhIL-12 & 80.24 & 16.9 & 42.04 & $16,703 \pm 2036$ \\
& SI & 1.7 & 1.1 & 1.2 & $16.6 \pm 2.4$ \\
IL-12p70 (pg/ml) & Medium & 0.9 & 17.1 & 0.16 & $2.7 \pm 0.8$ \\
& BCG & 49.8 & 64.8 & 77.5 & $75.8 \pm 7.8$ \\
& BCG + rhIFN- $\gamma$ & 219.1 & 272.1 & 410.7 & $700.1 \pm 89.9$ \\
& SI & 4.4 & 4.2 & 5.3 & $9.5 \pm 1.2$ \\
\hline
\end{tabular}

a Stimulation status: unstimulated (medium), stimulated with BCG alone, stimulated with BCG plus recombinant IL-12p70 (20 ng/ml), or stimulated with BCG plus recombinant IFN- $\gamma(5000 \mathrm{UI} / \mathrm{ml})$

SI stimulation index (BCG + cytokine/BCG), SEM standard error of the mean 


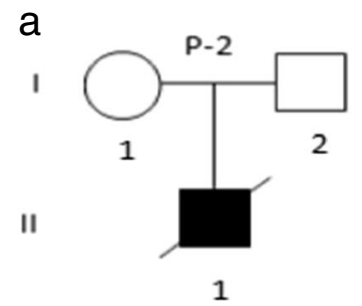

R283X

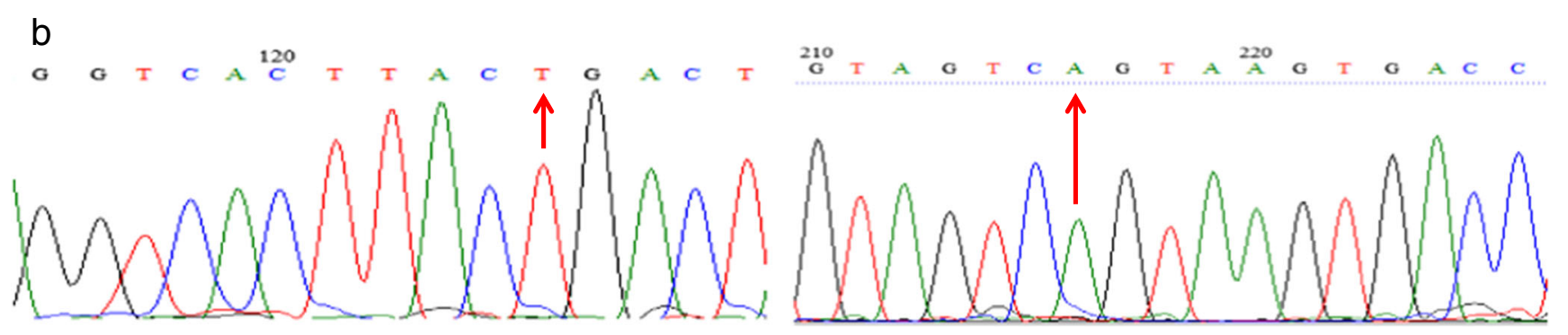

Fig. 2 Patient 2. a Pedigree of patient 2. b Sequencing analysis by dideoxy-nucleotide termination method. A mutation in exon 9 of the $I L 12 R B 1$ gene was found in this patient; left and right panels indicate results for forward and reverse sequencing

\section{Patient 3}

The third patient was a 7-year-old male vaccinated with BCG at birth that developed axillary lymphadenopathy at 5 months of age (Table 1; patient 3). The family had no history of TB or exposure to MTB. The patient was referred to the hospital due to an abscess in the thoracic cavity at 15 months associated with high fever, cough, and anemia and with abdominal ascites. Whole blood analysis gave the following results: WBC $3.9 \times 10^{3} / \mu \mathrm{l}$ (Neu 0\%; Lymph 48\%; Mono 49\%; Mix 3\%), $\mathrm{Hb} 6.3 \mathrm{~g} / \mathrm{dl}$, platelets $95 \times 10^{3} / \mu \mathrm{l}$, ESR $135 \mathrm{~mm} / \mathrm{h}$, and CRP $24 \mathrm{mg} /$ lit. BM aspiration was hypocellular. An evaluation of the abdominal cavity by imaging and by physical examination showed abdominal ascites, splenomegaly, and lymphadenopathy in the para-aortic, mesenteric, and celiac regions. A tuberculin skin test was negative and serum levels of $\operatorname{IgG}$ and $\operatorname{IgE}$ were elevated. GW was positive for mycobacteria on Lowenstein-Jensen medium. The lymphocyte transformation test (LTT) for BCG was 2.1 (normal levels $\geq 2.5$ ) and an NBT test was normal. Based on the physical analysis, laboratory data, and clinical signs, the patient underwent MSMD functional evaluation. A year and a half later, despite treatment with rifampin, isoniazid, ethambutol, and clarithromycin, the patient was re-admitted to the hospital due to backache, abdomen distension, and ascites. Radiologic analysis showed splenomegaly $(126 \mathrm{~mm})$. The ascites smear test was negative for AFB.

Four months later, the blood culture was positive for Kingella species and blood tests revealed the following results: WBC $7.05 \times 10^{3} / \mu \mathrm{l}$ (Neu 72.3\%; Lymph $10.1 \%$; Mono $11.9 \%$; Eos 5.4\%; Baso 0.3\%), Hb $12.4 \mathrm{~g} / \mathrm{dl}$, and PLT $241 \times 10^{3} / \mu \mathrm{l}$ and the $\mathrm{CD} 4 / \mathrm{CD} 8$ ratio was decreased compared to control levels (9/40). Serological assays and PCR for HIV were both negative. Thus, levofloxacin and IFN- $\gamma$ were added to the treatment regimen (rifampin, isoniazid, ethambutol, amikacin). However, at 5.5 years of age, the general condition of the patient deteriorated severely and the patient was again admitted to the hospital complaining of seizure and experienced respiratory distress, scrotal edema, severe backache, abdominal ascites, and distension during the hospitalization. Complete blood analysis gave the following results: WBC $6.8 \times 10^{3} / \mu \mathrm{l}$ (Neu 79\%; Lymph 13\%; Mix 8\%), Hb 10.7 g/dl, and PLT $142 \times 10^{3} / \mu \mathrm{l}$ and CRP was $61 \mathrm{mg} / \mathrm{l}$. A low liver function test ALT/AST ratio (15/20) was observed. Multiple brain abscesses and cerebritis were seen by MRI (Fig. 3a-c), probably due to BCG-osis, and peripheral edema in the cerebrium was observed by computed tomography. The BACTEC test result for ascites and TB PCR for synovial fluid were all negative for AFB and TB. One year later, the brain abscesses were PCR-positive for TB that was rifampin-resistant.

Progression from localized disease into disseminated infection suggested an underlying immunodeficiency and a genetic and functional analysis of the IL-12/IL-23/IFN- $\gamma$ loop was performed. Functional analysis revealed possible defects in the IL-12R $\beta 1$ (Table 2; patient 3 ) and genetic testing revealed the homozygous $I L 12 R B 1$ mutation c. $517 \mathrm{C}>\mathrm{T}$ in exon 5 (Fig. $3 e)$. This variation leads to an R173W amino acid change. Both parents of the patient were found to be heterozygous for this mutation. The R173W mutation has been previously reported in patients from Brazil, Venezuela, Poland (de Beaucoudrey et al. 2010), and Iran (Boisson-Dupuis et al. 2011). The previously reported Iranian patient had a history of PTB and cutaneous leishmaniasis (Boisson-Dupuis et al. 2011). To our knowledge, this is only the second patient in Iran reported with this mutation. 


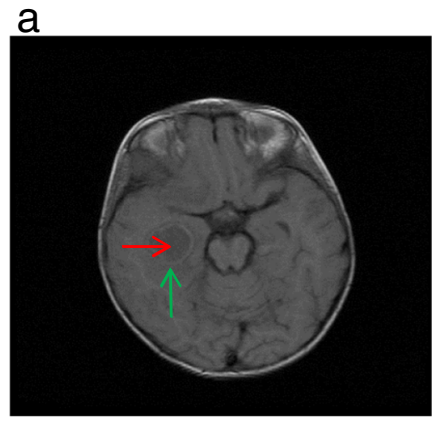

b

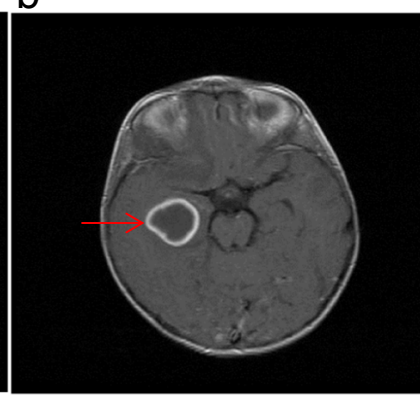

e

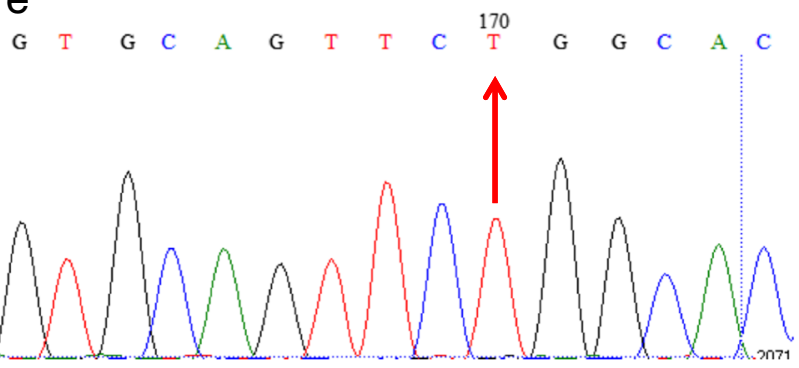

Fig. 3 Patient 3. Cerebral abscesses. a Axial T1-weighted spin MRI image without contrast. There is a hypo-intense lesion (red arrow) with an iso-intense capsule (green arrow) posterior to temporal horn of the right lateral ventricle. The lesion has compressed the temporal horn. b Axial T1-weighted spine MRI image with contrast. The capsule demonstrates marked enhancement (red arrow). c Axial T2weighted spine MRI image. The abscess is hyper-intense (red arrow).

\section{Discussion}

In young children, TB is often disseminated due to early, hematogenous spread of the Mycobacterium after primary pulmonary infection (Alcaïs et al. 2005). Clinical TB has now been described in a number of patients with IL-12/ IL-23/IFN- $\gamma$ system defects (Ottenhoff et al. 2005) and may result from autosomal recessive IL-12R $\beta 1$ deficiency, in at least some children (Boisson-Dupuis et al. 2011). The present study reports three Iranian children with complete IL-12R $\beta 1$ deficiency confirming previous studies in Iranian patients with an IL-12R $\beta 1$ defect (Azam Sarrafzadeh et al. 2016; Boisson-Dupuis et al. 2011; de Beaucoudrey et al. 2010; Fieschi et al. 2003; Parvaneh et al. 2015; Tabarsi et al. 2011).

In the present study, we screened for IL- $12 \mathrm{R} \beta 1$ mutations in suspected patients with MTB infection and reported three homozygous mutations: Q171P, R283X, and R173W. Patients 1 and 2 are the first reports of children with Mycobacterium infection due to IL-12R $\beta 1$ deficiency in Iran. Patient 3, with an R173W mutation, is only the second child reported in Iran with this particular mutation (Boisson-Dupuis et al. 2011) and MTB infection. Interestingly, all three of the IL-12R $\beta 1$-deficient patients reported here had been vaccinated with BCG and had developed BCG disease.
C

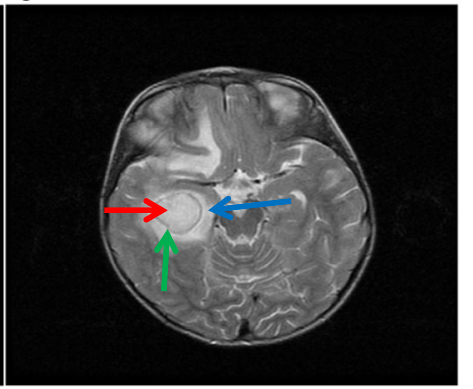

d

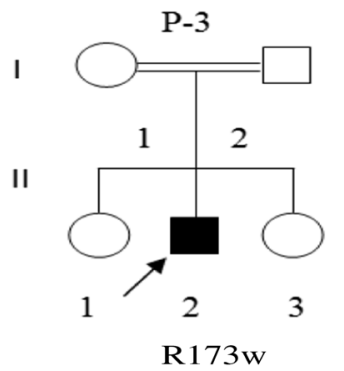

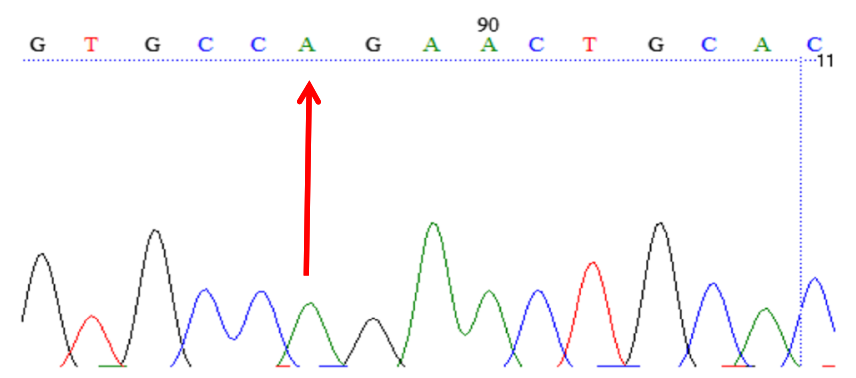

The capsule is iso-intense (green arrow) and the vasogenic edema surrounding the lesion (blue arrow) is also hyper-intense. d Pedigree of patient 3. e Sequencing analysis by dideoxy-nucleotide termination method demonstrated a mutation in exon 5 of the IL12RBI gene using forward (left panel) and reverse (right panel) sequencing
However, this deficiency is probably not unique to children, as a young adult case with TB and IL-12R $\beta 1$ deficiency has also been identified in Iran (Tabarsi et al. 2011). The increasing reports of Iranian patients with defects in the IL12/IL-23/IFN- $\gamma$ axis (Azam Sarrafzadeh et al. 2016; BoissonDupuis et al. 2011; de Beaucoudrey et al. 2010; Mansouri et al. 2005; Parvaneh et al. 2015), particularly in areas with a high prevalence of parental consanguinity and a very low prevalence of HIV (less than 0.15\%) (Boisson-Dupuis et al. 2011), suggest that screening for defects in this pathway should be considered in all cases. Thus, it will be important to define the prevalence of IL-12R $\beta 1$ deficiency and related disorders among children and adults with severe TB. This is especially important in countries where the disease is endemic such as Iran (Tabarsi et al. 2011).

Mandatory screening for IL-12R $\beta 1$ mutations that cause a defective IL-12/IL-23/IFN- $\gamma$ axis would not only improve the quality of patient care but also increase the safety monitoring for potential complications including infectious diseases in these subjects. A global registry of patients with genetic defects in the IL-12/IL-23/IFN- $\gamma$ axis such as that available at www.lovd.n1/IL12RB1 for IL-12Rß1 defects, and that are also available for most other genes that cause MSMD, together with awareness of these immunodeficiencies prior to $\mathrm{BCG}$ vaccination will improve the health and quality of care for these patients. 
Funding This study received financial support from National Research Institute of Tuberculosis and Lung Diseases (NRITLD), Shahid Beheshti University of Medical Sciences, Tehran, Iran.E .Mortaz was suported by National Institute for Medical Research Development (NIMAD) grant number 958274.

\section{Compliance with ethical standards}

Conflict of interest The authors declare that they have no conflict of interest.

Ethical approval Our study was performed according to the ethical standards issued by the COPE.

Informed consent Informed consent was obtained from all individual participants included in the study.

Open Access This article is distributed under the terms of the Creative Commons Attribution 4.0 International License (http:// creativecommons.org/licenses/by/4.0/), which permits unrestricted use, distribution, and reproduction in any medium, provided you give appropriate credit to the original author(s) and the source, provide a link to the Creative Commons license, and indicate if changes were made.

\section{References}

Alcaïs A, Fieschi C, Abel L, Casanova J-L (2005) Tuberculosis in children and adults. J Exp Med 202:1617-1621

Altare F et al (2001) Interleukin-12 receptor $\beta 1$ deficiency in a patient with abdominal tuberculosis. J Infect Dis 184:231-236

Azam Sarafzadeh S, Mahloojirad M, Nourizadeh M, Casanova J-L, Pourpak Z, Bustamante J, Moin M (2016) Mendelian susceptibility to mycobacterial disease due to IL-12R $\beta 1$ deficiency in three Iranian children. Iran J Public Health 45:249

Bogunovic D et al (2012) Mycobacterial disease and impaired IFN- $\gamma$ immunity in humans with inherited ISG15 deficiency. Science 337:1684-1688

Boisson-Dupuis S et al (2011) IL-12R $\beta 1$ deficiency in two of fifty children with severe tuberculosis from Iran, Morocco, and Turkey. PLoS One 6:e18524

Bustamante J et al (2011) Germline CYBB mutations that selectively affect macrophages in kindreds with X-linked predisposition to tuberculous mycobacterial disease. Nat Immunol 12:213-221

Caragol I et al (2003) Clinical tuberculosis in 2 of 3 siblings with interleukin-12 receptor $\beta 1$ deficiency. Clin Infect Dis 37:302-306

Casanova J-L, Abel L (2002) Genetic dissection of immunity to mycobacteria: the human model. Annu Rev Immunol 20:581-620

Casanova J-L, Jouanguy E, Lamhamedi S, Blanche S, Fischer A (1995) Immunological conditions of children with BCG disseminated infection. Lancet 346:581

Casanova J-L, Mansoori D, Fieschi C, Picard C, Dupuis S (2002) Inherited disorders of the interleukin-12-interferon gamma axis: Mendelian predisposition to mycobacterial disease in man. Tanaffos 1:9-25 de Beaucoudrey L et al (2010) Revisiting human IL-12R $\beta 1$ deficiency: a survey of 141 patients from 30 countries. Medicine 89:381

Falcão ACAM, Marques PTL, Santos AR, Oliveira JB (2012) Disseminated histoplasmosis caused by Il12rb1 gene mutations in two Brazilian siblings. J Clin Immunol 32:405

Feinberg J et al (2004) Bacillus Calmette Guérin triggers the IL-12/IFN- $\gamma$ axis by an IRAK-4-and NEMO-dependent, non-cognate interaction between monocytes, NK, and T lymphocytes. Eur J Immunol 34: 3276-3284

Fieschi C, Casanova JL (2003) Mini-review the role of interleukin-12 in human infectious diseases: only a faint signature. Eur J Immunol 33: 1461-1464

Fieschi C et al (2003) Low penetrance, broad resistance, and favorable outcome of interleukin 12 receptor $\beta 1$ deficiency. J Exp Med 197: $527-535$

Filipe-Santos O et al. Inborn errors of IL-12/23-and IFN- $\gamma$-mediated immunity: molecular, cellular, and clinical features. In: Seminars in immunology, 2006. vol 6. Elsevier, pp 347-361

Hambleton S et al (2011) IRF8 mutations and human dendritic-cell immunodeficiency. N Engl J Med 365:127-138

Haverkamp MH, van de Vosse E, van Dissel JT (2014) Nontuberculous mycobacterial infections in children with inborn errors of the immune system. J Inf 68:S134-S150

Lee W-I, Huang J-L, Yeh K-W, Jaing T-H, Lin T-Y, Huang Y-C, Chiu C$H$ (2011) Immune defects in active mycobacterial diseases in patients with primary immunodeficiency diseases (PIDs). J Formos Med Assoc 110:750-758

Mansouri D et al (2005) Inherited disorders of the IL-12-IFN- $\gamma$ axis in patients with disseminated BCG infection. Eur J Pediatr 164:753-757

Mortaz E et al (2015) Interaction of pattern recognition receptors with Mycobacterium tuberculosis. J Clin Immunol 35:1-10

Ottenhoff TH, Verreck FA, Lichtenauer-Kaligis EG, Hoeve MA, Sanal O, van Dissel JT (2002) Genetics, cytokines and human infectious disease: lessons from weakly pathogenic mycobacteria and salmonellae. Nat Genet 32:97-105

Ottenhoff TH, Verreck FA, Hoeve MA, van de Vosse E (2005) Control of human host immunity to mycobacteria. Tuberculosis $85: 53-64$

Özbek $N$ et al (2005) Interleukin-12 receptor $\beta 1$ chain deficiency in a child with disseminated tuberculosis. Clin Infect Dis 40:e55-e58

Parvaneh $\mathrm{N}$ et al (2015) Impaired in-vitro responses to IL-12 and IFN- $\gamma$ in Iranian patients with Mendelian susceptibility to mycobacterial disease. Allergol Immunopathol (Madr) 43:456-460

Prando C et al (2013) Inherited IL-12p40 deficiency: genetic, immunologic, and clinical features of 49 patients from 30 kindreds. Medicine 92:109

Reichenbach J, Rosenzweig S, Döffinger R, Dupuis S, Holland SM, Casanova J-L (2001) Mycobacterial diseases in primary immunodeficiencies. Curr Opin Allergy Clin Immunol 1:503-511

Schejbel L et al (2011) Combined IL-12 receptor and IgA deficiency in an adult man intestinally infested by an unknown, non-cultivable Mycobacterium. Scand J Immunol 74:548-553

Serour F, Mizrahi A, Somekh E, Feinberg J, Picard C, Casanova J-L, Dalal I (2007) Analysis of the interleukin-12/interferon- $\gamma$ pathway in children with non-tuberculous mycobacterial cervical lymphadenitis. Eur J Pediatr 166:835-841

Tabarsi P et al (2011) Lethal tuberculosis in a previously healthy adult with IL-12 receptor deficiency. J Clin Immunol 31:537-539 